\title{
Light path alignment for computed tomography of scattering material
}

\author{
Takafumi Iwaguchi ${ }^{1,2^{*}}$, Takuya Funatomi ${ }^{1}$, Hiroyuki Kubo ${ }^{1}$ and Yasuhiro Mukaigawa ${ }^{1}$
}

\begin{abstract}
We aim to estimate internal slice of the scattering material by Computed Tomography (CT). In the scattering material, the light path is disturbed and is spread. Conventional CT cannot measure the scattering material because they rely on the assumption that rays are straight and parallel. We propose light path alignment to deal with scattering rays. Each path of disturbed scattering light is approximated with a straight line. Then the light path in the object corresponding to a single incident ray is modeled as straight paths spreading from incident point. These spreading paths are aligned to be parallel, so that they can be used directly by conventional reconstruction algorithm.
\end{abstract}

\section{Introduction}

Measurement of the interior of an object is a key technique in various fields such as the inspection of food for finding foreign objects or the observation inside a human body.

$\mathrm{X}$-ray computed tomography $(\mathrm{CT})$ is one of those techniques that has been used in the inspection of the interior. However, the conventional X-ray CT is hard to use due to its cost, invasiveness, etc. Recently, optical measurement using visible (VIS)/near infrared (NIR) light is spotlighted because the optical system is inexpensive and safe compared to the X-ray.

However, optical measurement is challenging due to the scattering property of VIS/NIR light. In the case of the conventional $\mathrm{CT}$, the interior is estimated using a mathematical inverse transform based on an assumption that paths are straight and parallel. Therefore, the conventional $\mathrm{CT}$ is not directly applicable to optical measurement when the scattering occurred in the target material. For the scattering material, optical scattering tomography (OST) $[1,4,6]$ and optical diffusion tomography (ODT) [2, 3] have been proposed. In these methods, the paths of the scattered light are simulated and the interior is estimated from them. A drawback to these methods is the high computational cost due to simulating thousands of paths.

*Correspondence: iwaguchi.takafumi.il6@is.naist.jp

${ }^{1}$ Graduate School of Information Science, Nara Institute of Science and

Technology, Ikoma, Nara 630-0192, Japan

28916-5, Takayama, Ikoma, Nara 630-0192, Japan
In this paper, we propose an OST using the mathematical inverse transform. Figure 1 illustrates the overview of our proposed method. First, we propose a simplified model of the scattered light path. In this model, each path of the scattered light is approximated by a straight line. As a result, the paths in the object are regarded as a set of spreading lines from the incident point. We also propose light path alignment that converts these spreading paths into parallel paths. This alignment makes it possible to estimate interior using mathematical inverse transform that has been used in the conventional ray $\mathrm{CT}$, although the light is scattered.

\section{Method}

\subsection{Conventional CT method}

To begin with, we describe how the interior is estimated in the conventional CT. The conventional X-ray CT has been used for decades because it provides a clear visualization of the interior of the object. The conventional CT relies on the important X-ray's property that it passes straight through the object without reflection and refraction, but it is attenuated a certain rate depending on the material.

Figure 2a illustrates a setup of the conventional CT measurement. We call this measurement parallel transmission measurement. A parallel beam is cast onto an object from the light source, and its projection on the plane of the opposite side is observed. This measurement is executed from various views.

When the paths in the object are straight and parallel, the interior is reconstructed using the inverse Radon

\section{Springer}




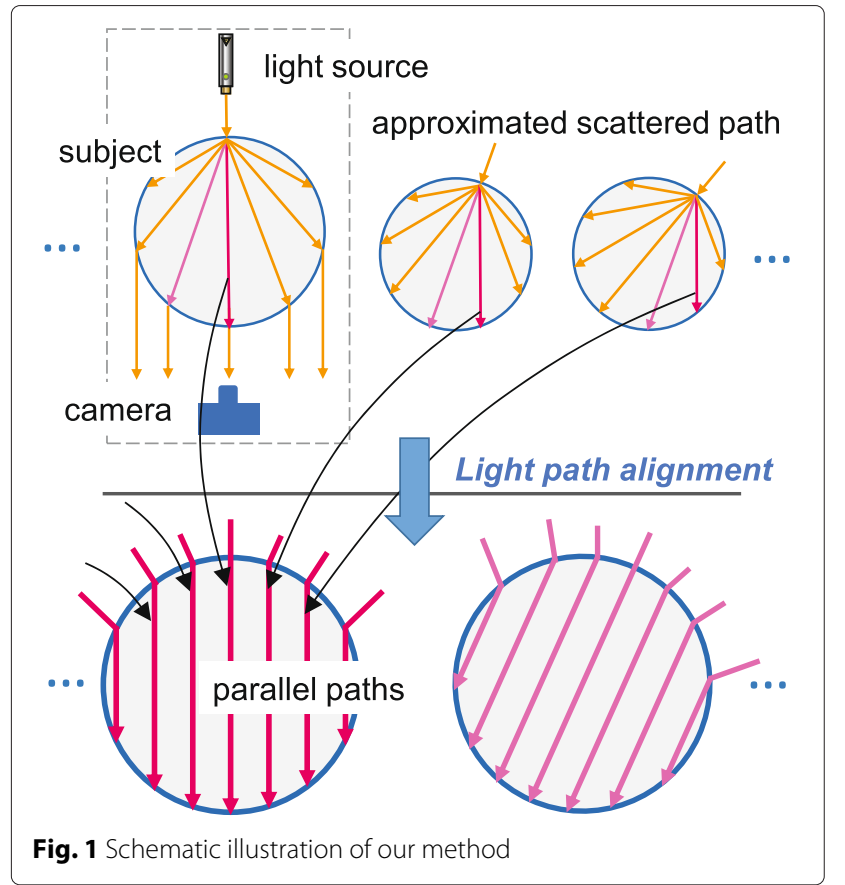

transform. When a single ray is cast onto the object, it travels in a straight path. Its intensity is attenuated according to the path length and the absorption rate of the material. A projection of a single ray is regarded as the integral of the absorption over the straight line. The inverse Radon transform will give a relationship between the projection data obtained from the measurement and the spatial distribution of the absorption rate. Filtered back projection (FBP) [5] is an implementation of the inverse Radon transform, which is superior in terms of accuracy and speed.

\subsection{Model of path of scattered rays}

In the case of optical measurement, the interior cannot be estimated in the same way since the light path does not

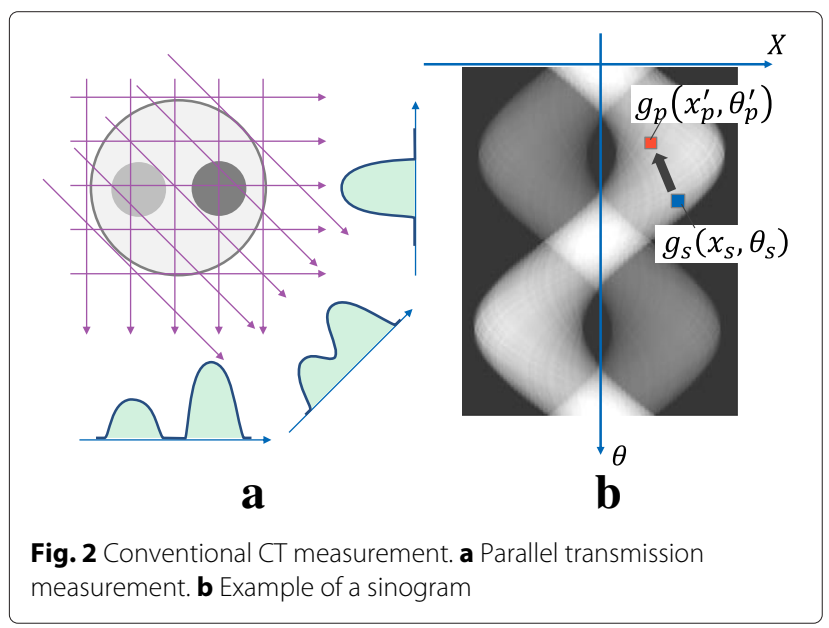

follow the assumption in the parallel transmission measurement. Now, we describe the model of the paths of the scattered light that our $\mathrm{CT}$ method relies on.

Limiting incident ray In order to apply the conventional $\mathrm{CT}$ for recovering the interior, points on the surface where the ray entered and where the ray went out must be determined first.

Figure 3 illustrates the paths of the scattered light when the parallel beam is cast. When each ray enters a scattering material, the path is scattered into multiple and spreading paths. We cannot determine where the ray that reached on the specific point comes from because the rays from all incident rays reach the same point on the surface. To deal with this problem, we cast a single ray on the object at observation so that the incident point is determined.

Approximating path of scattered light Figure 4a illustrates the paths of the scattered light corresponding to a single incident ray. In order to simplify the disturbed path of the scattered light, we approximate each path of the scattered light by a shortest path connecting the incident point and the point on the surface where the light reached as shown in Fig. 4b. Then the paths in the object are regarded as a set of spreading straight rays from the incident point.

According to this model, we propose shortest-path transmission measurement to estimate the interior of the scattering object. The left-top figure of Fig. 1 illustrates the setup of our measurement. A single ray is cast toward an arbitrary point of the object, and the object's surface is measured using a camera. Then measurement is repeated

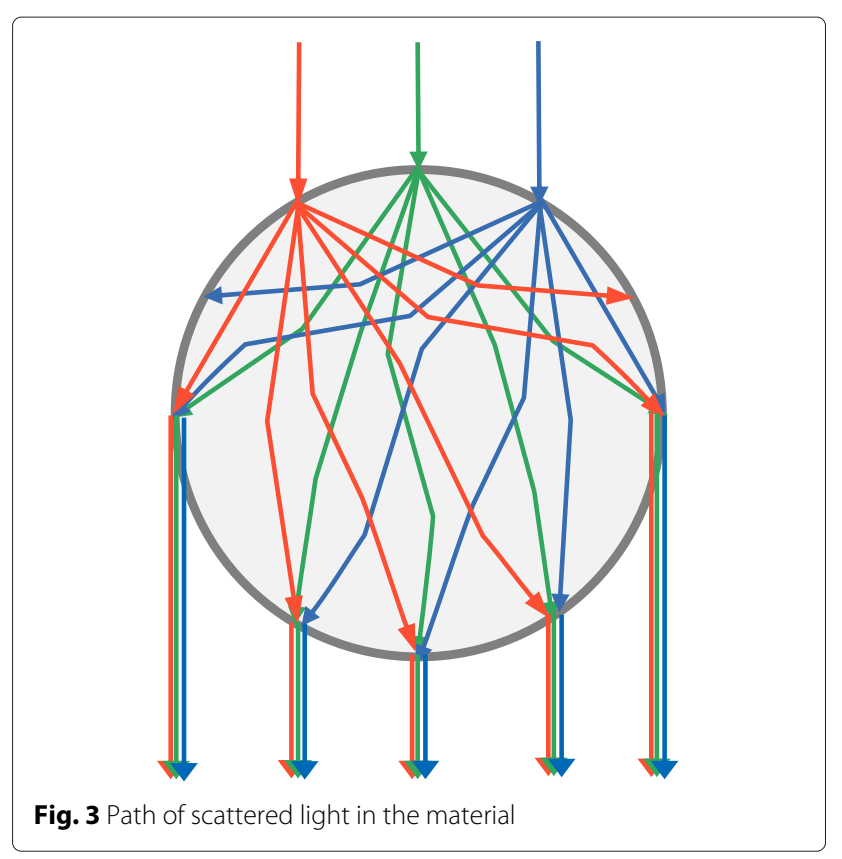




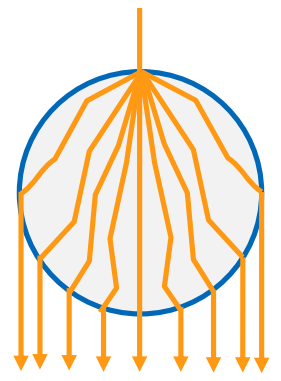

a

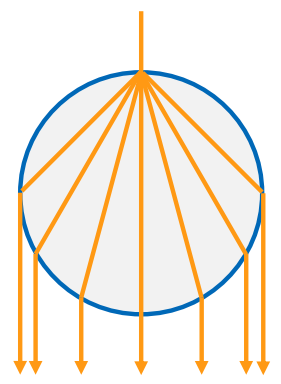

b

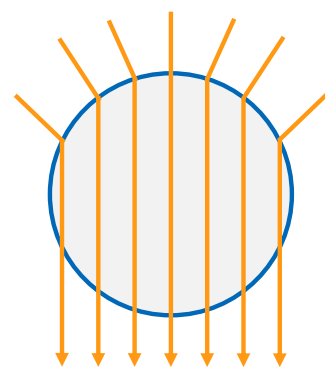

c

Fig. 4 Model of the light path. a Actual path of scattered light. b Shortest-path transmission model. c Parallel paths obtained by light path alignment

with rotating the object. As a result, sets of spreading straight rays for various angles of the incident rays are captured.

\subsection{Light path alignment}

According to the light path model, the paths in the object are simplified as spreading straight rays. However, it is still insufficient to apply inverse Radon transform because they are not parallel. Here, we introduce light path alignment that converts these simplified paths to parallel paths.

A schematic illustration of light path alignment is shown in Fig. 1. The light path alignment converts paths of the scattering surface measurement shown in the first row into the parallel paths in the second row. From a single measurement, scattered light through the object is observed and it is treated as a set of spreading straight paths from the incident point. Other sets of the paths are observed by casting rays from different directions. Then we pick up the paths of the same direction from these sets and align them according to the location so as to be parallel as shown in Fig. 4c.

Light path alignment can be implemented as a conversion of sinogram. Sinogram stores rays observed by a measurement as shown in Fig. 2b. Suppose that a $u v$ coordinate is fixed on the object and the camera and the light source rotates around the origin of the $u v$ coordinate as shown in Fig. 5. Let $X$ be the location where a ray reaches on the projection plane and $\theta$ be the angle between the orientation of the camera and $v$ axis. In the sinogram, a ray reaches $X$ of the projection plane of the camera stored at $(X, \theta)$.

We denote a sinogram of shortest-path transmission measurement by $g_{s}$ and one of parallel transmission measurement by $g_{p}$. Light path alignment is a conversion from $g_{s}$ to $g_{p}$.

In order to formulate a conversion between both sinograms, we discuss about the relationship between the coordinates of the sinogram and the path of a ray. In the case of parallel transmission measurement as shown in Fig. $5 \mathrm{a}$, a ray that reaches $x_{p}$ of the projection plane with

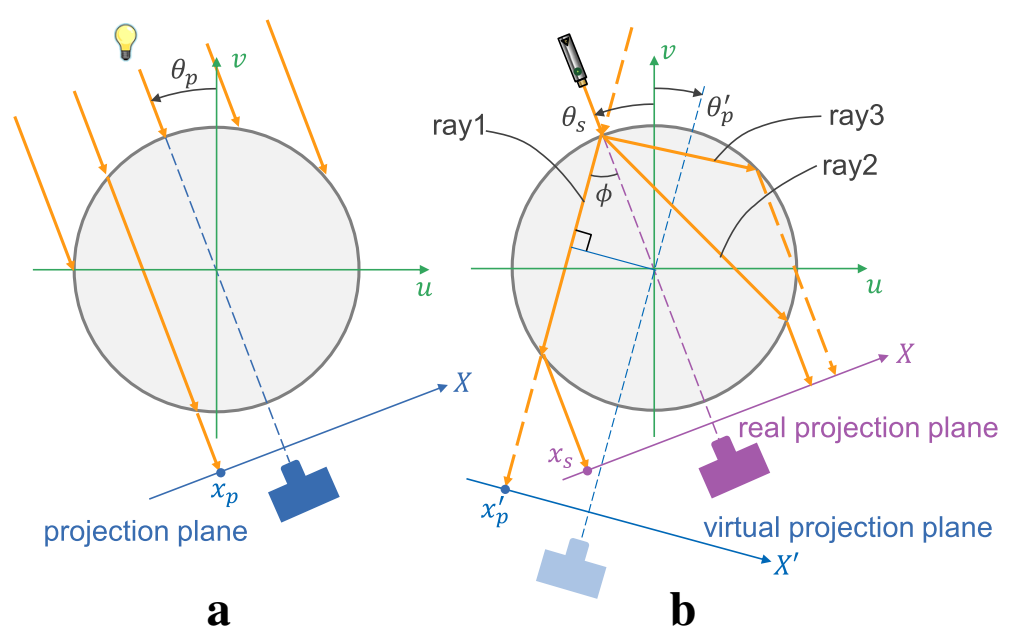

Fig. 5 Geometry of measurement. a Parallel transmission measurement. b Shortest-path transmission measurement 
$\theta=\theta_{p}$ is stored at $g_{p}\left(x_{p}, \theta_{p}\right)$. In the case of shortestpath transmission measurement as shown in Fig. 5b, "ray 1 " that reaches $x_{s}$ of the projection plane with $\theta=\theta_{s}$ is stored at $g_{s}\left(x_{s}, \theta_{s}\right)$. We focus on the path of this ray. If this path is extended through the surface, it can be regarded as a ray of parallel transmission measurement with a virtual projection plane $X^{\prime}$ and $\theta=\theta_{p}^{\prime}$. Let $x_{p}^{\prime}$ be a location that the extended path reaches, then the ray is stored at $g_{p}\left(x_{p}^{\prime}, \theta_{p}^{\prime}\right)$ in the sinogram of the converted sinogram. From the discussion above, light path alignment is altered by moving $g_{s}\left(x_{s}, \theta_{s}\right)$ to $g_{p}\left(x_{p}^{\prime}, \theta_{p}^{\prime}\right)$ like as shown in Fig. 2 . This conversion is possible when each coordinate in the sinogram $g_{p}$ represents a unique ray in the object.

We formulate the relationship between $\left(x_{p}^{\prime}, \theta_{p}^{\prime}\right)$ and $\left(x_{s}, \theta_{s}\right)$ in the case of the object is a cylinder and the camera model is orthogonal projection. Let $\phi$ be an angle between the ray from the light source and the ray in the object. The relationship between $\left(x_{p}^{\prime}, \theta_{p}^{\prime}\right)$ and $\left(x_{s}, \theta_{s}\right)$ is formulated as follows:

$$
\begin{aligned}
x_{p}^{\prime} & =\frac{x_{s} \cos \phi}{\sin 2 \phi}=\frac{x_{s}}{2 \sin \phi} \\
\theta_{p}^{\prime} & =\phi-\theta .
\end{aligned}
$$

Therefore, the sinogram of the shortest-path transmission measurement $g_{s}$ can be converted to one of the parallel transmission measurement $g_{p}$ by the following equation:

$$
g_{p}\left(x_{p}, \theta_{p}\right)=g_{s}\left(\frac{x_{s}}{2 \sin \phi}, \phi-\theta_{s}\right) .
$$

The paths of the scattered light are converted to parallel paths by this conversion, then the optical CT for the scattering object using inverse Radon transform is possible.

\subsection{Optimal placement of light source}

By light path alignment, the paths of shortest-path transmission measurement are converted to parallel. However, there are some unobserved paths due to the incompleteness of the measurement. For example, "ray 2" in Fig. $5 \mathrm{~b}$ is observed because it reaches the surface that can be seen from the camera. In contrast, "ray 3" cannot be observed because it reaches the surface that is hidden from the camera. Therefore, it is necessary to find the optimal setup of measurement to minimize the number of unobservable paths.

In the discussion so far, the light source is placed at the opposite of the camera. However, a number of the unobserved paths changes corresponding to the angle between the light source and the camera. Assume the camera is fixed; now, we derive an optimal placement of the light source relative to the camera.

Figure 6 illustrates the observable area in the case the object is a cylinder and the camera model is orthogonal projection. We denote the angle between the orientation of the light source and the orientation of the camera by $\theta_{l}$. The rays that reach the surface highlighted by green are observable by the camera, and the area that these rays pass through is shown in blue. It is found that there are areas that are unobservable from the camera.

In order to evaluate these areas, we take a look at a distance between a ray and the center of the object. Let the slice of the cylinder a unit circle and $d$ the distance between the ray and the center of the object. In the case of parallel transmission measurement, $d$ takes a range $0 \leq d \leq 1$. In the case of shortest-path transmission measurement, the range depends on $\theta_{l}$. We denote the range of $d$ by $d_{\min } \leq d \leq d_{\max }$. For $0^{\circ} \leq \theta_{l} \leq 90^{\circ}$, the rays that pass near the surface are unobservable. $d_{\min }$ and $d_{\max }$ are given as follows:
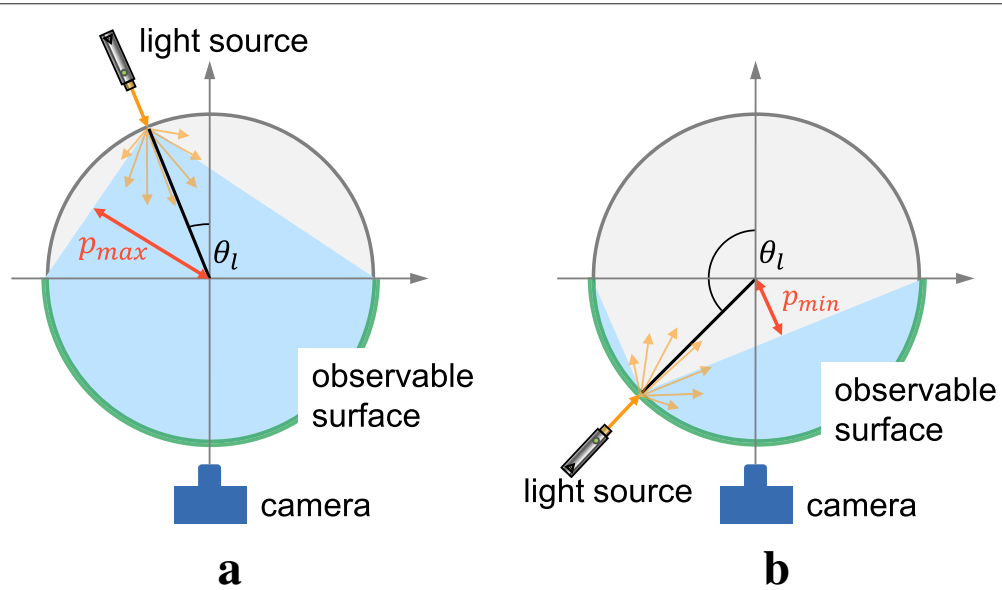

Fig. 6 Observed paths corresponding to a placement of the light source. $\mathbf{a} 0^{\circ} \leq \theta_{l} \leq 90^{\circ}$. b $90^{\circ} \leq \theta_{l} \leq 180^{\circ}$ 


$$
\begin{aligned}
& d_{\min }=0 \\
& d_{\max }=\cos \left(\frac{\pi}{4}-\frac{\theta_{l}}{2}\right) .
\end{aligned}
$$

For $90^{\circ} \leq \theta_{l} \leq 180^{\circ}$, the rays that passes around the center are unobservable. $d_{\min }$ and $d_{\max }$ are given as follows:

$$
\begin{aligned}
& d_{\min }=\sin \left(\frac{\theta_{l}}{2}-\frac{\pi}{4}\right) \\
& d_{\max }=1 .
\end{aligned}
$$

We define the coverage of the observation as a ratio of the range taken by $d$ compared to the radius. Let $p$ denote the coverage of the observation; it is calculated as follows:

$$
p=d_{\max }-d_{\min } .
$$

The coverage can be comprehended as the area of a sinogram. The distance $d$ corresponds to the $\mathrm{x}$-axis of the sinogram because $x$-axis of the sinogram is a displacement of a ray relative to the center of the object. Therefore, $d_{\min }, d_{\max }$, and $p$ mean distances are shown in Fig. 7a. The coverage will be the ratio of the area filled up in the sinogram. Figure $7 \mathrm{~b}$ shows the coverage of observation with respect to $\theta_{l}$. It is found that the coverage takes its maximum 1 at $\theta_{l}=90^{\circ}$. This means the complete paths are observable for this setting; thus, it is the optimal placement of the light source.

\section{Experiment}

We performed the numerical simulation for the evaluation of the proposed method. We confirmed that our method estimates the interior and confirmed the improvement of reconstruction by using the optimal measurement setup.

As a synthetic data, a distribution of the absorption rate is given as shown in the second row of Fig. 8a with the dimensions of $64 \times 64$. The first row of Fig. 8a shows the ideal sinogram obtained by the parallel transmission measurement. It is found that traces of the circles appear as two sinusoidal curves.

First, we show the sinogram when the scatter occurs and the reconstructed interior from it using FBP without alignment. The projection was simulated with the assumption of the ideal shortest-path transmission model where the scattering at surface is isotropic and the path of the ray in the object is straight. We performed an experiment for $\theta_{l}=0^{\circ}$ where the coverage is not complete and for $\theta_{l}=90^{\circ}$ where the measurement setup is optimal. Figure $8 \mathrm{~b}$ shows the sinogram and the reconstructed interior for $\theta_{l}=0^{\circ}$. Although the sinogram consists of two curves reflecting the absorption corresponding to two circles, they are not perfect sinusoids like in the sinogram of parallel transmission measurement in Fig. 8a. The reconstructed interior consists of two blurred shapes. The circles were not reconstructed correctly because the light paths were not aligned. Figure $8 \mathrm{c}$ shows the sinogram and the reconstructed interior for $\theta_{l}=90^{\circ}$. In this case, the light was cast perpendicularly to the orientation of the camera from the left side; thus, traces appear mainly on the right side. The reconstructed interior is totally unclear due to the corrupted shape of the sinogram.

Next, the sinogram was converted by light path alignment. Figure $8 \mathrm{~d}$ shows the aligned sinogram and the reconstructed interior for $\theta_{l}=0^{\circ}$. Two sinusoidal curves appear after the light path alignment is executed. However, the sinogram is lacking its both sides since the measurement setup is not optimal. In the reconstructed interior, the shapes of the circles are reconstructed. We can see that ellipse-shaped artifacts appeared and the shape outside the artifacts is unclear. These artifacts are considered to be caused by the incompleteness of the sinogram. Figure $8 \mathrm{e}$ shows the aligned sinogram and the reconstructed interior for $\theta_{l}=90^{\circ}$. The aligned sinogram is complete and is substantially identical with the sinogram of parallel transmission measurement. The

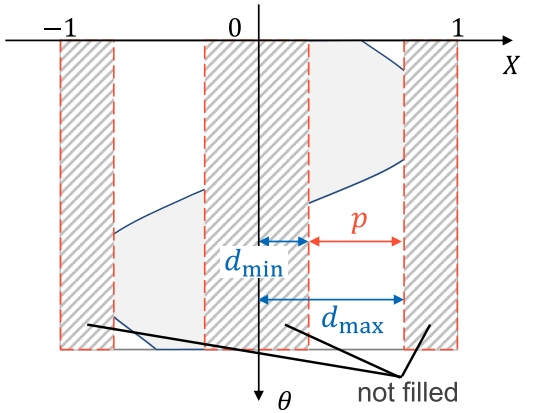

$\mathbf{a}$

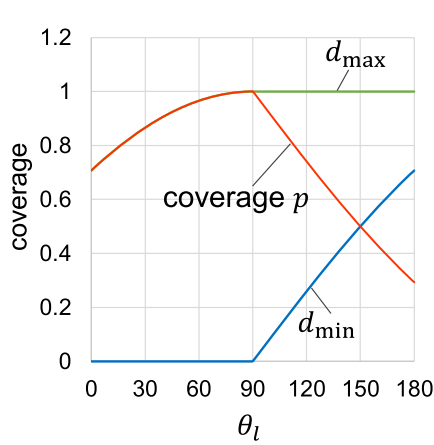

b

Fig. 7 Coverage of the measurement. a Coverage as an area in the sinogram. $\mathbf{b}$ Coverage vs the angle of light 


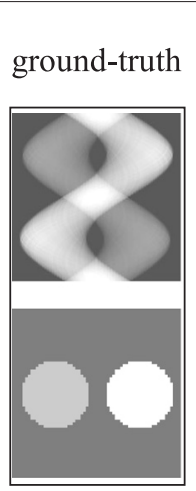

a

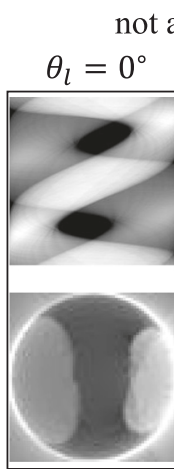

b not aligned

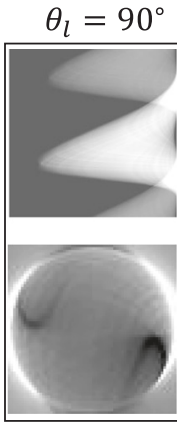

c

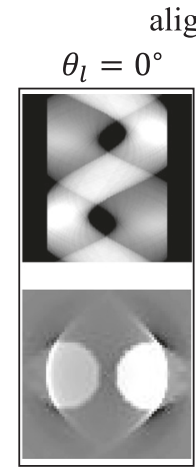

d aligned

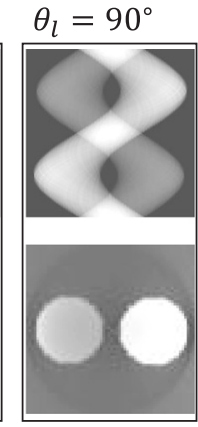

e

Fig. 8 Sinogram and reconstructed interiors. a Ground-truth of the distribution. b, c The reconstructed interior from the original sinogram where $\theta_{l}=0^{\circ}$ and $\theta_{l}=90^{\circ} . \mathbf{d}, \mathbf{e}$ The reconstructed interior from the aligned sinogram where $\theta_{l}=0^{\circ}$ and $\theta_{l}=90^{\circ}$

complete observation of rays was possible because all the ray through the closer half to the camera reach to the observable surface in this case. In the reconstructed interior, we can see the interior is reconstructed correctly.

\section{Conclusion}

We have proposed an OST using inverse mathematical transform. In order to reconstruct the interior using the inverse Radon transform, the paths in the object have to be parallel and straight. We have proposed shortest-path transmission model that approximates the paths of the scattered light as spreading straight lines from the incident point. We have also proposed light path alignment that converts approximated paths to parallel and optimal placement of the light source.

From the numerical experiments, we have presented the interior is reconstructed correctly by our proposed method. We have also shown that the reconstruction is improved by choosing the optimal placement of the light source.

For future work, we plan to measure a real object using our method.

\section{Competing interests}

The authors declare that they have no competing interests.

\section{Authors' contributions}

TI carried out the implementation and drafted the manuscript. TF, HK, and YM were advisors and helped to draft the manuscript. All authors read and approved the final manuscript.

\section{Acknowledgements}

This work was partly supported by JSPS KAKENHI Grant Numbers JP26700013, JP15K16027.

Received: 28 April 2016 Accepted: 17 June 2016

Published online: 02 August 2016

\section{References}

1. Akashi R, Nagahara H, Mukaigawa Y, Taniguchi R-i (2015) Scattering tomography using ellipsoidal mirror. In: 2015 21st Korea-Japan Joint Workshop on Frontiers of Computer Vision (FCV). pp 1-5

2. Durduran T, Choe R, Baker WB, Yodh AG (2010) Diffuse optics for tissue monitoring and tomography. Rep Prog Phys 73(7):076701

3. Hebden JC, Gibson A, Austin T, Yusof RM, Everdell N, Delpy DT, Arridge SR, Meek JH, Wyatt JS (2004) Imaging changes in blood volume and oxygenation in the newborn infant brain using three-dimensional optical tomography. Phys Med Biol 49(7):1117

4. Ishii Y, Arai T, Mukaigawa Y, Tagawa J, Yagi Y (2013) Scattering tomography by monte carlo voting. IAPR Int Conf Mach Vis Appl 1:1-5

5. Shepp L, Logan B (1974) The Fourier reconstruction of a head section. IEEE Trans Nucl Sci 21(3):21-43

6. Tamaki T, Yuan B, Raytchev B, Kaneda K, Mukaigawa Y (2013) Multiple-scattering optical tomography with layered material. The 9th International Conference on Signal-Image Technology \& Internet-Based Systems (SITIS):93-99

\section{Submit your manuscript to a SpringerOpen ${ }^{\circ}$ journal and benefit from:}

- Convenient online submission

- Rigorous peer review

- Immediate publication on acceptance

- Open access: articles freely available online

- High visibility within the field

- Retaining the copyright to your article

Submit your next manuscript at $\boldsymbol{s p r i n g e r o p e n . c o m ~}$ 